Article

\title{
Modeling of Geometric Change Influence on Blast-Wave Propagation in Underground Airways Using a 2D-Transient Euler Scheme
}

\author{
Liang Wang ${ }^{1, *}$, Sisi Que ${ }^{2}$, Jerry C. Tien ${ }^{3}$ and Nassib S. Aouad ${ }^{4}$ \\ 1 State Key Lab of Coal Mine Disaster Dynamics and Control, Chongqing University, \\ Chongqing 400044, China \\ 2 School of River and Ocean Engineering, Chongqing Jiaotong University, Chongqing 400074, China; \\ sq3g3@mst.edu \\ 3 Department of Civil Engineering, Monash University, Clayton Campus, Wellington Road, \\ Clayton, VIC 3800, Australia; Jerry.Tien@monash.edu \\ 4 Mining and Nuclear Engineering Department, Missouri University of Science and Technology, \\ Missouri, MO 65409, USA; narzf@mst.edu \\ * Correspondence: lw38c@cqu.edu.cn; Tel.: +86-23-6510-4951
}

Academic Editor: David Cliff

Received: 24 March 2016; Accepted: 5 July 2016; Published: 3 August 2016

\begin{abstract}
The impact of methane explosions on mining operations can never be over-emphasized. The safety of miners could be threatened and local ventilation facilities are likely to be damaged by the flame and overpressure induced by a methane explosion event, making it essential to understand the destructiveness and influence range of a specific explosion. In this paper, the attenuation effect of geometric changes, most commonly bends, obstacles, and branches, present in the way of blast-wave propagation and the capability of the selected numerical model were studied. Although some relevant experimental research has been provided, quantitative analysis is insufficient. This paper investigates the attenuation factors of seven bends, three obstacles, and two T-branch scenarios to ascertain a better insight of this potentially devastating event quantitatively. The results suggest that (1) the numerical model used is capable of predicting four of the seven validated scenarios with a relative error less than $12 \%$; (2) the maximum peak overpressure is obtained when the angle equals $50^{\circ}$ for bend cases; and (3) the selected numerical scheme would overestimate the obstacle cases by around $15 \%$.
\end{abstract}

Keywords: methane deflagration; bend; obstacles; Attenuation Factor; transient Euler method

\section{Introduction}

A methane explosion is one of the most violent mine accidents which could result in a high death toll and devastating financial losses. In 1906, 1099 miners were killed in the Courrieres Coal Mine explosion in France. In December 1907, the Monongah Numbers Six and Eight explosions in West Virginia, USA, claimed 362 lives, which was the worst American mine disaster. Moreover, in May 1928, 195 miners were killed in the Mather Number One mine explosion in Pennsylvania, USA. The most catastrophic explosion ever recorded was the Honkeiko Colliery disaster of 1942, in China, in which 1549 miners lost their lives [1]. Despite the attention to mining safety brought by methane explosions, accidental deaths in the coal industry continued through the mid-20th Century. For example, in December 1951, a methane explosion in Orient Number Two Mine, Illinois, resulted in 119 fatalities. From 1900 to 2010, 10,390 miners lost their lives in 420 explosions in the USA alone. Methane explosions continue to be the number one killer amongst all mining accidents [2].

The major destructiveness of methane explosions result from the extremely high overpressure and temperature during an event. It includes rapid combustion reactions when the methane/air mixture is 
ignited by an unexpected energy release, such as sparks, electrical arc, or lightning in a confined space. A transition from laminar to turbulent combustion enables a positive feedback process which leads to obvious flame acceleration and extremely high blast overpressure [3-5]. Geometric changes on an airway could have a significant impact on the propagation of the blast-wave induced by a methane explosion. The influences should be understood and quantified when investigating an explosion in an underground ventilation system (airway network). The most representative geometric changes in longwall and room-and-pillar airway systems are bends, obstacles, and branches [3]. These geometric changes' influences have been investigated experimentally under different experimental conditions and qualitative results were provided [6-8]. Some distinctive studies demonstrated that the bend of a duct would result in two opposite effects on the overpressure produced by an explosion. It could increase the overpressure if it is located in a gas-filled region and otherwise attenuates it $[9,10]$. Branching of a duct and sudden area increase in cross-sections will decrease the flame speed induced by the methane explosion, as well as overpressure [11]. The impacts of obstacles are similar to bends; hence, the presence of obstacles in a methane-filled site could increase overpressure, but decreases it during blast-wave propagation [8]. In summary, qualitatively speaking, geometric changes at an explosion site will either accelerate the flame and strengthen the overpressure or attenuate the blast-wave which passes through them. In this article, a numerical model using an unsteady 2D compressible Euler scheme has been developed and aims to characterize and quantify these influences. A series of lab-scale explosion experiments have also been conducted to validate the model and will be introduced in the next section.

\section{Experiment}

\subsection{Experimental Instruments}

The main components of the experiment system belong to Gas Explosion Lab of China University of Mining and Technology (CUMT), Xuzhou, China. This experiment design is based on the experimental framework constructed by CUMT. In this research, five units are included. There are igniter, explosion duct, gas bag, gas valve, and a data collection system (Figure 1). The explosion duct is an $80 \mathrm{~mm} \times 80 \mathrm{~mm}$ square cross-section steel duct which can withstand the maximum of $20 \mathrm{MPa}$ overpressure. The data collection system (model: CS20182-32 with 32 Channels, $20 \mathrm{MHz}$ collection frequency, and 1 bit accuracy) (CUMT) is connected to a personal computer which is used to record experimental results.

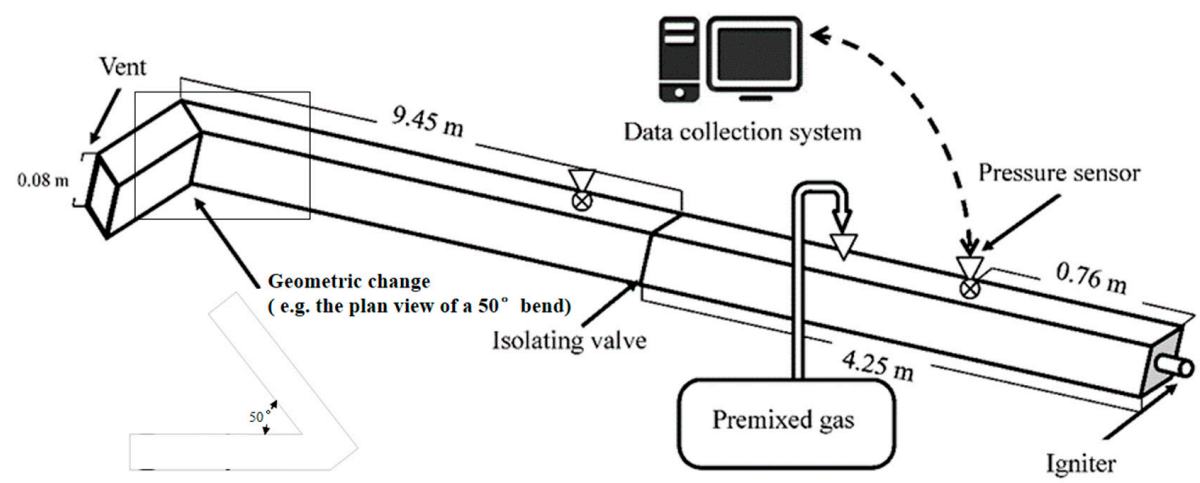

Figure 1. Schematic of the methane explosion experiment system (not to scale).

\subsection{Experiment Progress}

As shown in Figure 1, the methane explosion experiment system has five main components and the experiment process consists of the following steps. At the beginning, the desired methane concentration $(9.5 \%)$ is prepared in the gas bag. Opening of the valve between the gas bag and explosion duct will feed prepared gas into the explosion duct, which was vacuumed by a vacuum pump beforehand. After that, ignition of the explosive mixture triggers an explosion while opening the 
gas valve between the gas-filled region and the following duct that opens to atmosphere. An explosion will last for several to several hundred milliseconds when the overpressure and flame propagation are captured by photosensitive flame and pressure sensors (10 mV/V output). Meanwhile, these signals will be collected and organized by the data collection system and stored in a database in the computer.

\subsection{Scenarios}

In this study, a detailed plan for the experiment is developed to characterize methane explosion characteristics under different geometric configurations. The methane concentration of $9.5 \%$ was chosen to represent the stoichiometric condition. Under this concentration, seven groups of tests were conducted with and without geometrical changes. The geometric changes tested include four major types of bends with the bending angles of $50^{\circ}, 90^{\circ}, 120^{\circ}$, and $140^{\circ}$; and obstacles with three blockage-ratios of $25 \%, 50 \%$, and $75 \%$. Tests were repeated with an "acceleration spiral" used to characterize the explosion in airways with obstructions within the gas-filled section. The flame could be accelerated due to a further stretching of the flame front in the vicinity of the spiral [12]. The results of the experiments with the presence of an acceleration spiral will not be included, but will be used as a reference to the $2 \mathrm{D}$ numerical simulations. Each scenario was repeated three times to increase accuracy. For detailed instruction of experimental instruments and procedure please refer to our previous work [13].

\subsection{Experimental Results}

The maximum recorded overpressures upstream and downstream of a selected geometric change in each prescribed scenarios are listed in Table 1.

Table 1. Experimental overpressures and Attenuation Factors $(\mathrm{Pa} / \mathrm{Pa})$.

\begin{tabular}{cccc}
\hline Bends and BRs & Upstream $\left(\mathbf{P}_{\mathbf{0}}\right) / \mathbf{P a}$ & Downstream $\left(\mathbf{P}_{\mathbf{1}}\right) / \mathbf{P a}$ & $\boldsymbol{\eta}^{*}$ \\
\hline $50^{\circ}$ Bending & 90,604 & 50,098 & 1.726 \\
$90^{\circ}$ Bending & 43,037 & 41,284 & 1.131 \\
$120^{\circ}$ Bending & 213,920 & 304,181 & 0.703 \\
$140^{\circ}$ Bending & 76,571 & 85,128 & 0.945 \\
BR 25\% & 99,981 & 90,871 & 1.1 \\
BR 50\% & 93,003 & 70,116 & 1.326 \\
BR 75\% & 33,224 & 18,878 & 1.759 \\
$* \eta$ refers to the attenuation factor defined by $\eta=\mathrm{P}_{0} / \mathrm{P}_{1}$ [6]. BR represents the blockage ratio.
\end{tabular}

As shown by the experimental attenuation factor $(\eta)$ for bends in Table 1 , this factor is observed to be inversely proportional to bending, with ranges between $0^{\circ}$ and $120^{\circ}$. The blockage effect is more obvious in smaller angles, and the attenuation factor drops below one in bending angles of $120^{\circ}$ and $140^{\circ}$, before it increases back to one at $180^{\circ}$. The greatest $\eta$ value of 1.726 is found at a $50^{\circ}$ bend, since the blast-wave can hardly go through it, and reflections are also constrained by this geometry.

For obstacles with different blockage-ratios (BR), the trend of the attenuation factor change is simpler than those found in bends, it increases with the BR monotonically. The greatest $\eta$ recorded is 1.759 obtained when the BR is equal to $75 \%$. The observation suggests that larger blockage ratios tend to attenuate a blast-wave more significantly. Future experimental investigation needs to be conducted for more cases having a wider range of bending angles, which is beyond the scope of this research.

\section{Numerical Modeling}

The modeling of methane explosions is based on conservation equations which are known as governing equations in fluid mechanics. In a methane explosion, Mach number can reach as high as 2 [14]. The variation of density should be taken into account. The compressibility adds considerable complexities to the work. Therefore, assumptions are required to simplify the simulation. Based on the two-region theory, the explosion is divided into two relatively independent sections. In this study, only the pure blast-wave region will be simulated [15]. All of the reaction in the flame-dominated 
region (Figure 2) will not be considered. Instead, the boundary conditions of scenarios will be assigned by experimental results. The simulations seek to repeat the experiment and validate the modeling.

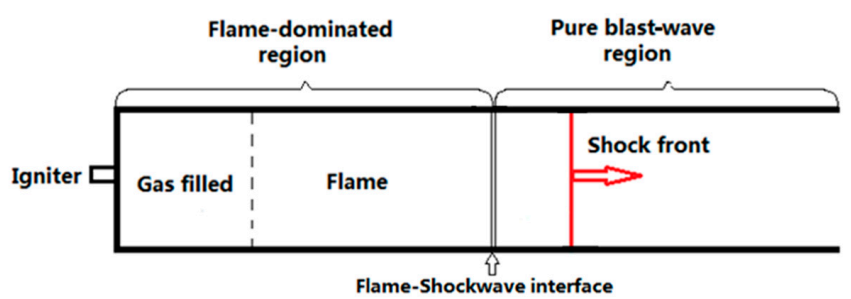

Figure 2. Regions of the methane explosion process in a duct [15].

Governing Equations. In the numerical studies of the driver section, 3D Navier-Stokes (NS) conservation equations, turbulence, and chemical reactions were used. However, when attempting to model a blast-wave propagating through a geometric change, a 2D Euler model is considered appropriate if turbulence and reaction are no longer factors. The flow should be considered as both transient and compressible since a blast-wave propagation is highly time dependent. According to the above assumptions, the trimmed equations used in this study are shown as the following:

$$
\begin{gathered}
\frac{\partial \rho}{\partial t}+\nabla(\rho u)=0 \\
\frac{\partial(\rho u)}{\partial t}+\nabla(\rho u \mathbf{u})=-\frac{\partial P}{\partial x} \\
\frac{\partial(\rho u)}{\partial t}+\nabla(\rho v \mathbf{u})=-\frac{\partial P}{\partial y} \\
\frac{\partial(\rho e)}{\partial t}+\nabla(\rho e \mathbf{u})=\frac{\partial Q}{\partial t}+\nabla(Q \mathbf{u})-\left(\frac{\partial q}{\partial x}+\frac{\partial q}{\partial y}\right) \\
P=\rho R T
\end{gathered}
$$

The equation system above are called Euler equations, and are assumed to be inviscid. Note that in the Euler equations, the viscous terms in momentum equations are eliminated due to turbulent shear being ignored in the blast-wave section. The unsteady terms are retained to capture the transient nature of the blast-wave propagation. All of the terms in the $z$ direction are also eliminated due to the 2D assumption.

Density Based Solver. The density-based method was used for simulations including geometric changes. A density-based solution is an alternative to the pressure-based solution employed by ANSYS Fluent (ANSYS, Canonsburg, PA, USA). It solves Equations (1) to (4) simultaneously in vector form [16]. The advantage is its ability to resolve transient flows when density changes significantly with respect to time, such as blast-wave propagation. Specifically, the primitive flow parameters are solved by the following four steps listed below:

(a) To update the primitive flow parameters from the last solution or given initial conditions;

(b) To solve the continuity, momentum, and energy equations simultaneously to get flow parameters for the next time step;

(c) To use the updated flow parameters to solve separate scalars if appropriate; and

(d) To check the convergence of the solution

Another feature of this numerical scheme is the usage of an explicit temporal scheme. An explicit scheme is different from the implicit schemes used by the 3D and 1D models, respectively. Its expression can be shown as: 


$$
\phi^{n+1}=\phi^{n}+\Delta t F\left(\phi^{n}\right)
$$

where the superscripts represent the time level belonging to a flow parameter. $n$ means the current time step, while $n+1$ means the next time step.

Meshing. To model the blast-wave propagation through a geometric change, geometrical models should be developed and meshed (spatially discretized) before implementing a numerical simulation. The pre-process software ANSYS Gambit version 2.4.6 (ANSYS) is used to develop the geometric models and to mesh them. Unstructured quadrilateral meshing is used in all bends except for the $90^{\circ}$ geometric change, which requires a quadrilateral mapping structural mesh. For the obstacles and T-branches, quadrilateral mapping is used.

Numerical Details. After the meshing, the governing equations can be solved in the discretized domains. The CFD (Computational Fluid Dynamics) general code ANSYS Fluent Cortex version 14.5.0 was employed as the solver. A density-based solution was used in all selected scenarios. Explicit schemes were used to discretize the computational domain and the time duration as well. Courant number (CFL) is set to be one to ensure that numerical calculations are stable for an explicit temporal discretization. A least squares cell-based method is used for gradient treatment and third-order monotonic upstream-centered scheme for conservation laws (MUSCL) method is used for primitive flow parameters. The time step size can be calculated by Equation (7) given CFL equal to 1 .

$$
\Delta t=\frac{C F L \cdot \Delta x}{|V|+c}
$$

where $V$ is characteristic flow velocity magnitude $c$ is sound of speed, and $\Delta x$ is characteristic cell size.

CFD calculations were conducted using computer with a quad-core i7 3770K and 16 GB RAM. Two parallel processors were used. The residuals are generally at the $10^{-1}$ level.

Geometry and Initial Condition. In this study, the blast-wave passing through twelve different geometrical considerations are simulated. The portion of the experimental explosion duct with the geometric changes is selected to be the computational domain of the model. The length of the arms of each bend is $0.35 \mathrm{~m}$. Their diameter (width of the duct) is $0.08 \mathrm{~m}$. The pressure sensor upstream is placed a distance of $0.12 \mathrm{~m}$ from the inlet, along the centerline of the duct. The sensor downstream the bend is placed $0.12 \mathrm{~m}$ from the outlet, along the duct centerline.

The experiment results are assigned in corresponding locations to maintain consistency, where upstream pressure sensors are situated. For example, in the T-branch case, if the measurement of the eleventh overpressure sensor (P11) of a methane explosion is $0.05 \mathrm{MPa}$, the region in red will be assigned as $0.05 \mathrm{MPa}$ overpressure throughout this region (Figure 3). The inlet (the left boundary) will be considered as the pressure inlet, which has a constant overpressure of $0.05 \mathrm{MPa}$. Constant pressure of one atm $(0.101325 \mathrm{MPa})$ is imposed to the outlet boundaries at the top and right ends. The scenarios selected are considered as supersonic flows with the average Mach number of the shock front upstream of the geometric change as 1.35. This setup is another simplification from a real blast-wave to an ideal shockwave. A blast-wave will decay after its wave front, while a shockwave does not. Under this assumption, the predicted overpressure will be relatively higher and more sustainable than the real case. The simulation results of all twelve cases will be demonstrated as follows.

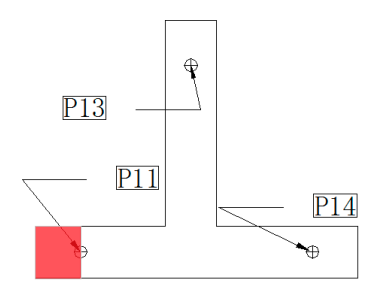

Figure 3. Pressure sensor layout and high overpressure region. 


\section{Simulation Results}

Simulation results processed by CFD-post imbedded in ANSYS are provided below. There are two types of demonstrations, pressure gradient contour and overpressure history. The former presents the blast-wave front location and high pressure regions; the latter illustrates the pressure variations on the two selected monitor surfaces (Figure 4a).
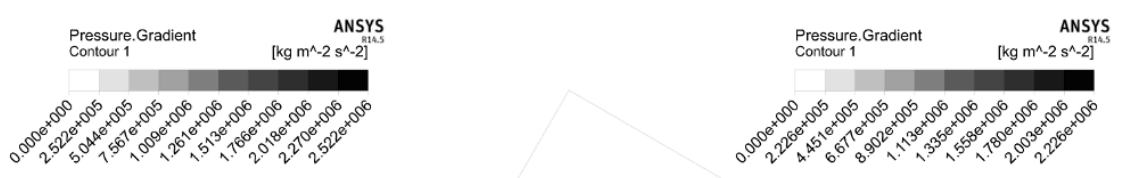

II

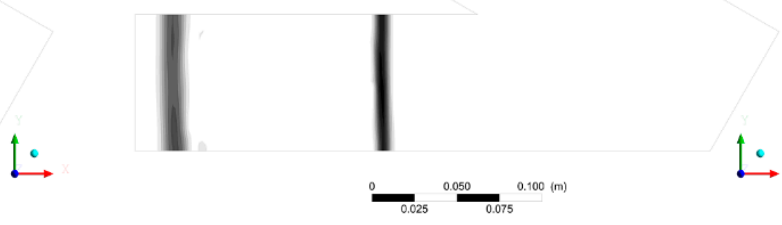

(a)

(b)

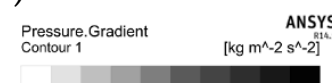

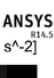
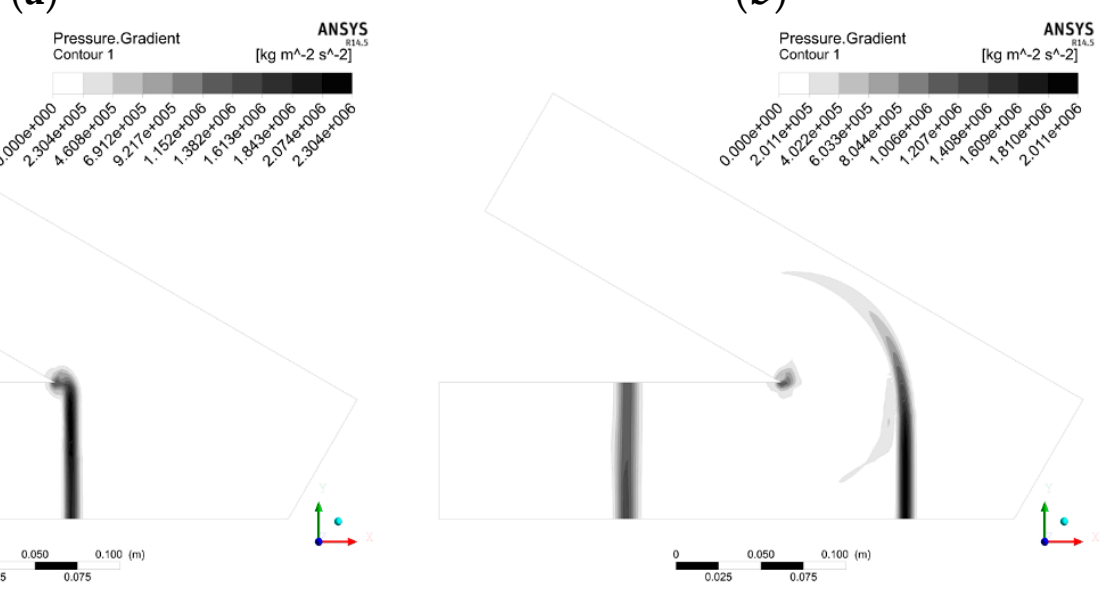

(c)

(d)

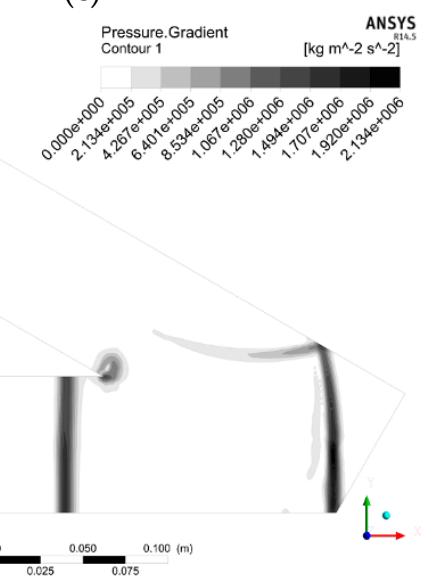

(e)

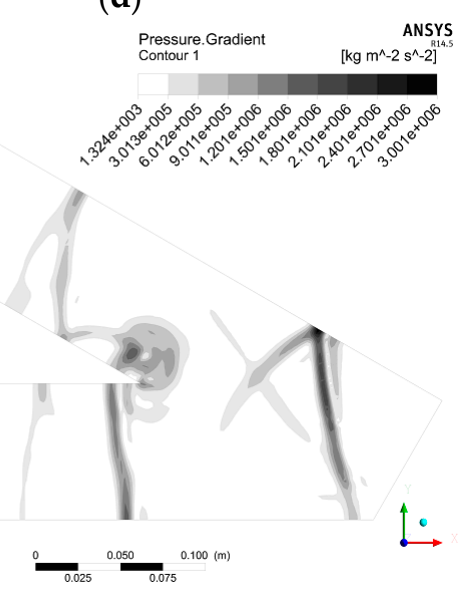

(f)

Figure 4. Overpressure gradient contours for a blast-wave propagating through a $30^{\circ}$ bend after (a) 25 time steps; (b) 100 time steps; (c) 200 time steps; (d) 300 time steps; and at (e) $0.000682 \mathrm{~s}$ and (f) $0.00137 \mathrm{~s}$. 


\subsection{Bends}

Comparing the four bend scenarios in the experiments, seven angles; i.e., $30^{\circ}, 40^{\circ}, 50^{\circ}, 90^{\circ}, 120^{\circ}$, $140^{\circ}$, and $160^{\circ}$, are selected in the numerical study. Their overpressure contours during propagation and overpressure histories, upstream and downstream of the bend, will be demonstrated.

$30^{\circ}$ Bend. The predicted overpressure gradient contours after 25, 100, 200, and 300 time steps when the blast-wave is passing through the $30^{\circ}$ bend are shown in Figure 4a-d. Figure 4e,f illustrate the overpressure contour at 0.000682 and $0.00137 \mathrm{~s}$ when maximum overpressure of the first impulse is obtained by the upstream and downstream sensors, respectively. The convergence is normally of a magnitude of $10^{-1}$ for the continuity equation, and $10^{-3}$ for other equations.

The overpressure histories upstream and downstream of the bend are demonstrated in Figure 5. The maximum overpressure for the first impulse upstream the bend is $24,616 \mathrm{~Pa}$ at $0.000682 \mathrm{~s}$, while the attenuated maximum overpressure for the first impulse downstream the bend is $21,400 \mathrm{~Pa}$ at $0.00137 \mathrm{~s}$, the attenuation factor $\eta$ is 1.15 .

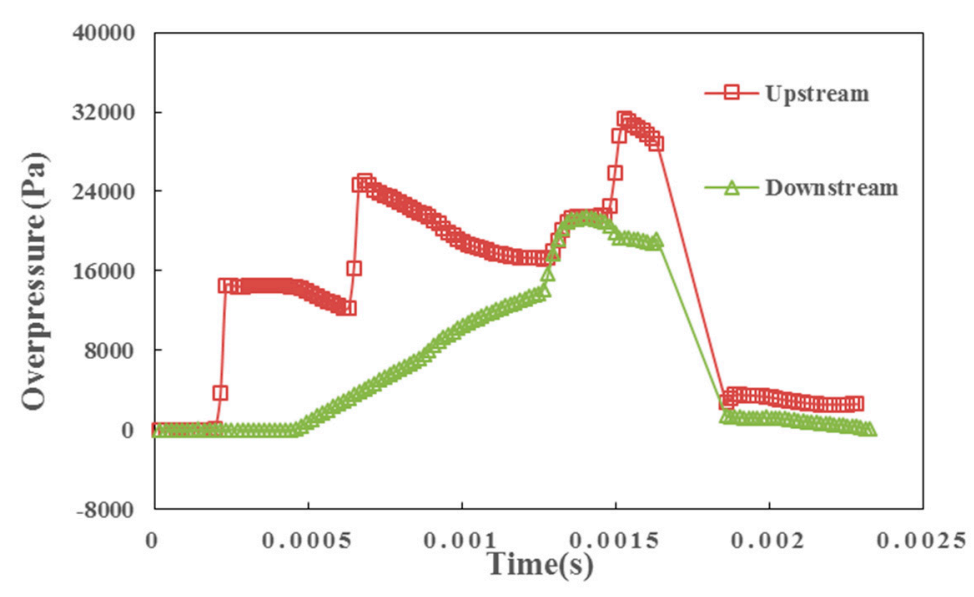

Figure 5. Overpressure histories upstream and downstream of the $30^{\circ}$ bend.

As illustrated in Figures 4 and 5, the maximum overpressure of shock-wave recorded is obtained when the incident wave front is passing through the upstream pressure sensor (at $0.000682 \mathrm{~s}$ ). At this instant, the total energy has not been dissipated by the interaction by being reflected in the vicinity of the bending corner. A high overpressure gradient region is observed within the outer corner of the bend where the shockwave is reflected (Figure 4e) and on the tip of the sharp angle where a vortex start to develop (Figure $4 \mathrm{~d}$ ). The presence of diffraction-reflection interaction leads to a pressure increase downstream of the bend. The shock retained a relatively high level for less than $0.0005 \mathrm{~s}$ until a sudden decline at $0.0017 \mathrm{~s}$. This decrement of overpressure shown in Figure 5 of both upstream and downstream sensors is due to the shock front having gone through the duct.

Other Bends. Similar to the $30^{\circ}$ bend, blast-wave propagation over time and the overpressure histories of two monitor surfaces are used for analyzing the attenuation effect of the other six bend cases. Figures $6-11$ show the process of a blast-wave passing through the explosion duct, predicting overpressure gradient contours after 25, 100, 200, and 300 time steps and at the instance of the maximum overpressures of the bending scenarios of $40^{\circ}, 50^{\circ}, 90^{\circ}, 120^{\circ}, 140^{\circ}$, and $160^{\circ}$ were obtained by upstream and downstream sensors. 


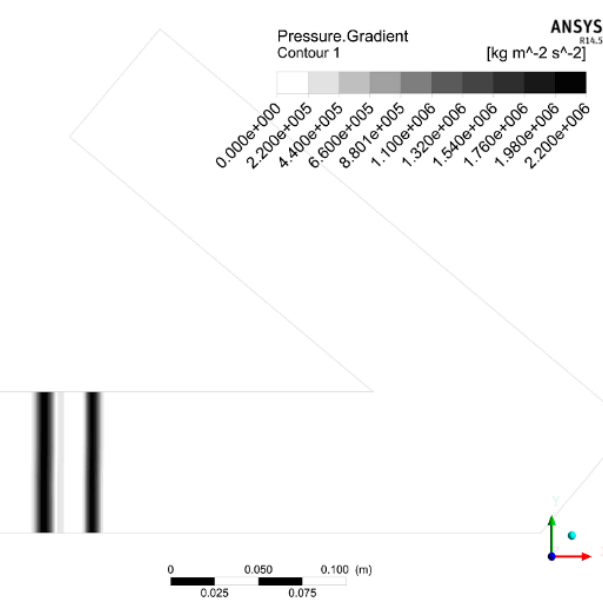

(a)
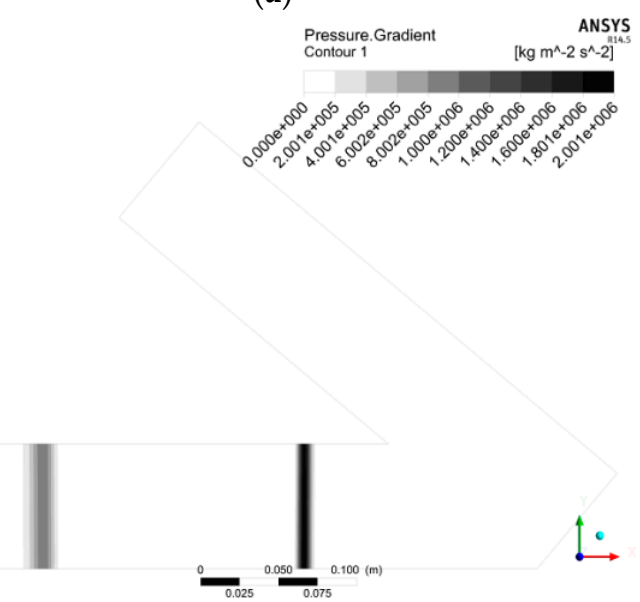

(c)

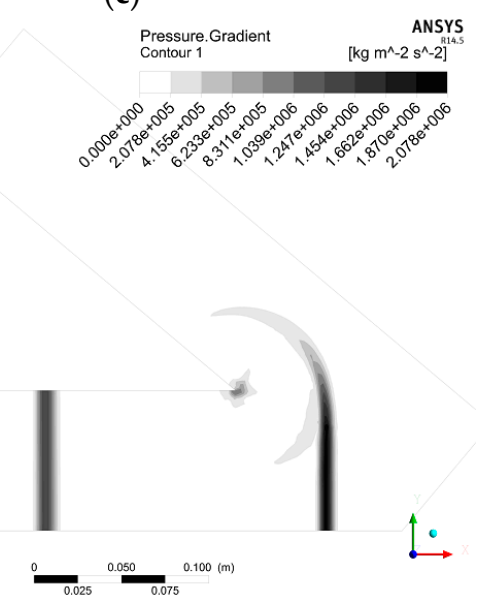

(e)
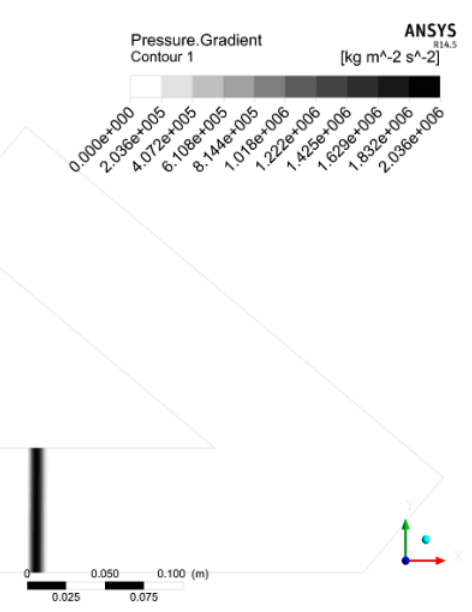

(b)
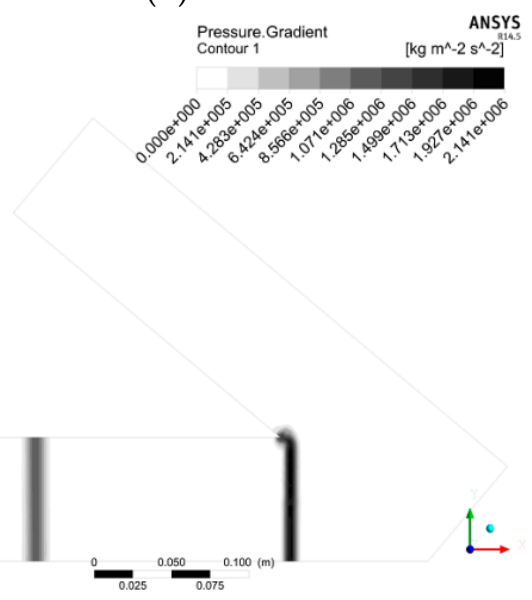

(d)

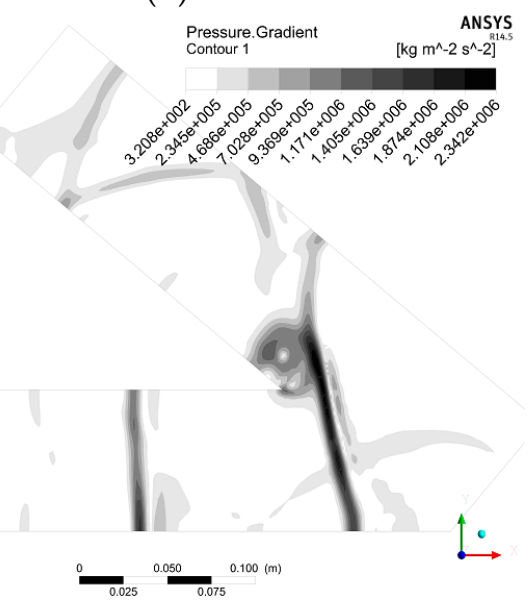

(f)

Figure 6. Overpressure gradient contours for blast-wave propagating through a $40^{\circ}$ bend at (a) 25 time steps, (b) 100 time steps, (c) 200 time steps, (d) 300 time steps; and at (e) $0.000691 \mathrm{~s}$ and (f) $0.00158 \mathrm{~s}$. 


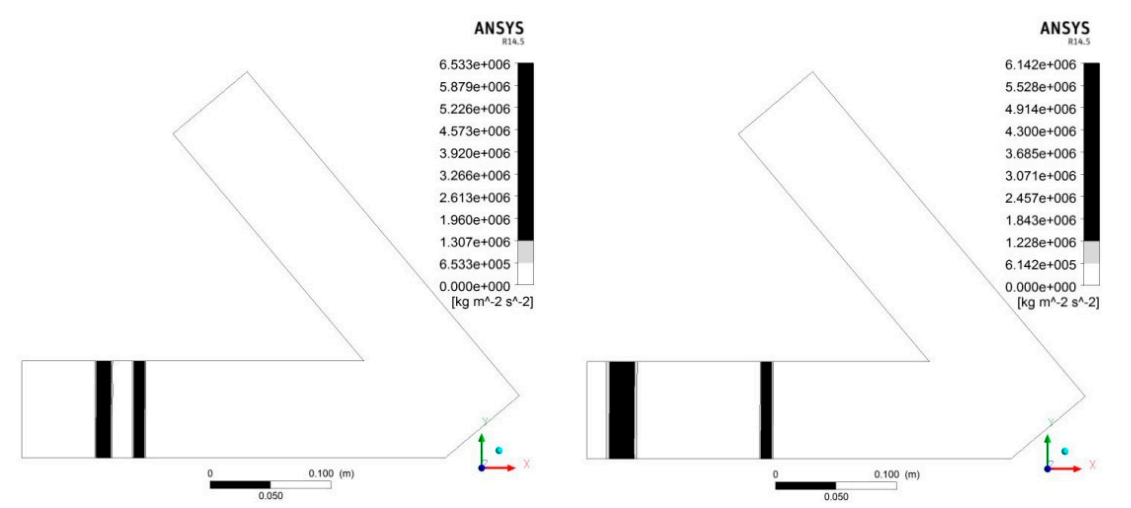

(a)

(b)

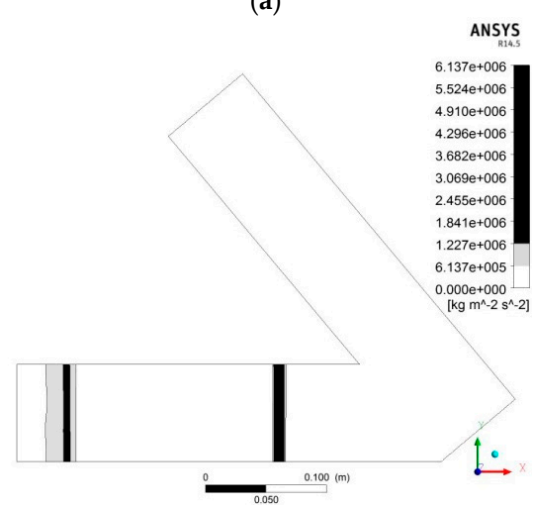

(c)
ANSYS

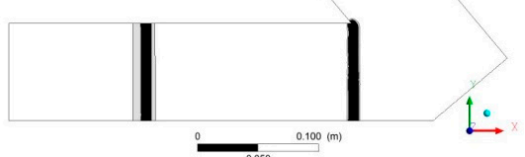

(d)

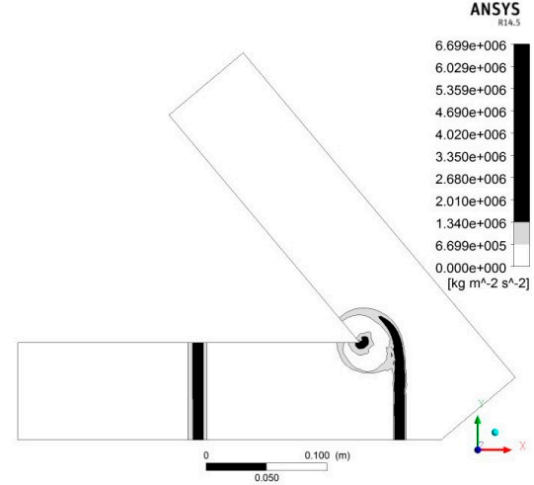

(e)

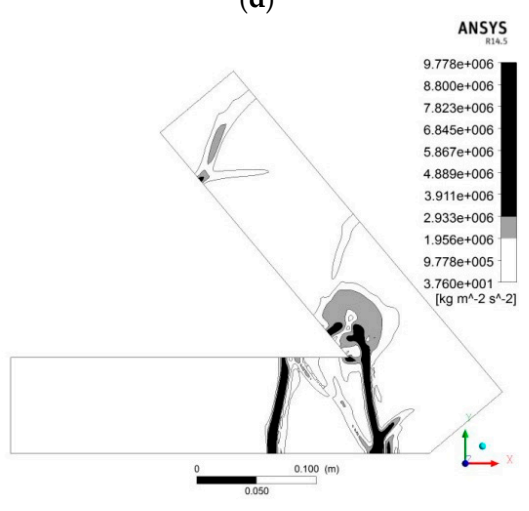

(f)

Figure 7. Overpressure gradient contours for blast-wave propagating through $50^{\circ}$ bend at (a) 25 time steps; (b) 100 time steps; (c) 200 time steps; (d) 300 time steps; and at (e) 0.000595 s and (f) 0.00149 s.

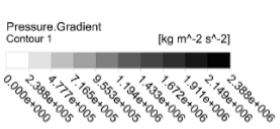

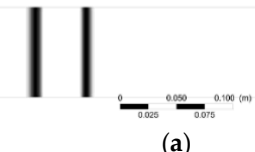

ANSYS

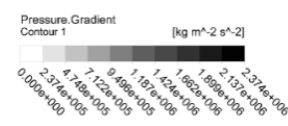

$\stackrel{1}{\circ}$

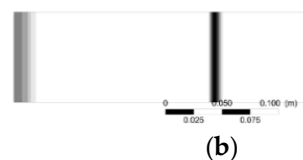

ANSYS

$\stackrel{\circ}{\circ}$

Figure 8. Cont. 


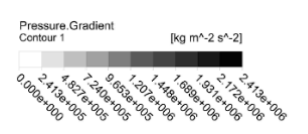

ANSYS

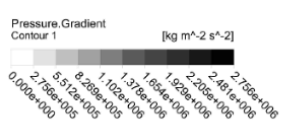

ANSYS
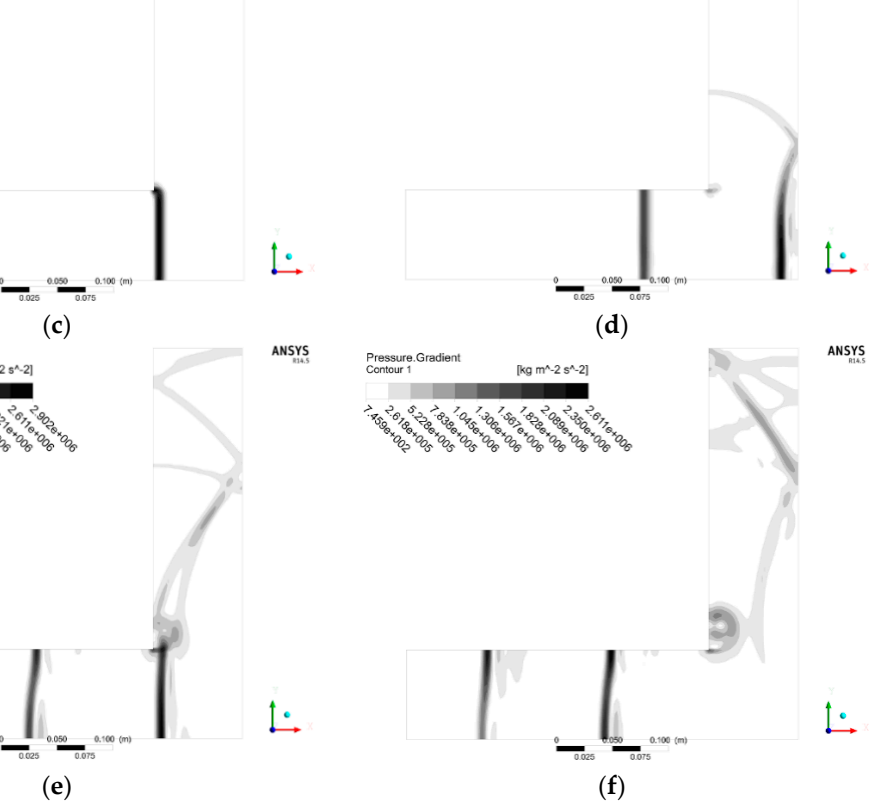

Figure 8. Overpressure gradient contours for blast-wave propagating through $90^{\circ}$ bend after (a) 25 time steps; (b) 100 time steps; (c) 200 time steps; (d) 300 time steps; and at (e) $0.00136 \mathrm{~s}$ and (f) $0.00161 \mathrm{~s}$.

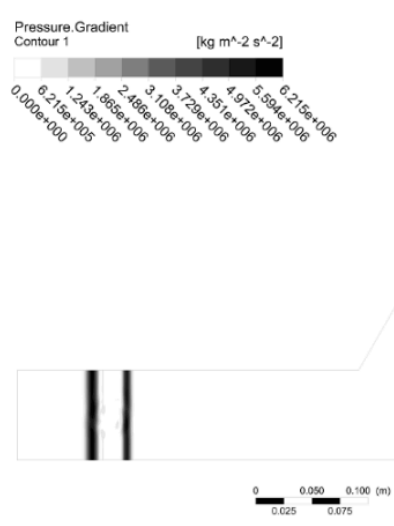

(a)
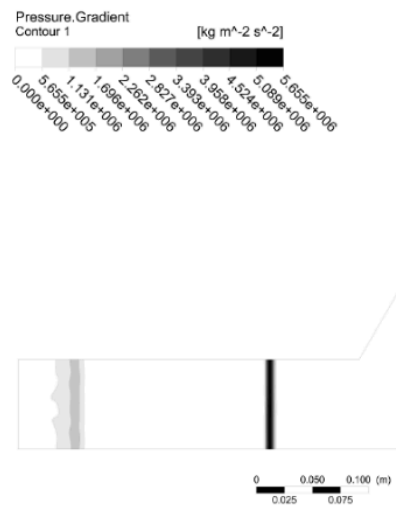

(c)
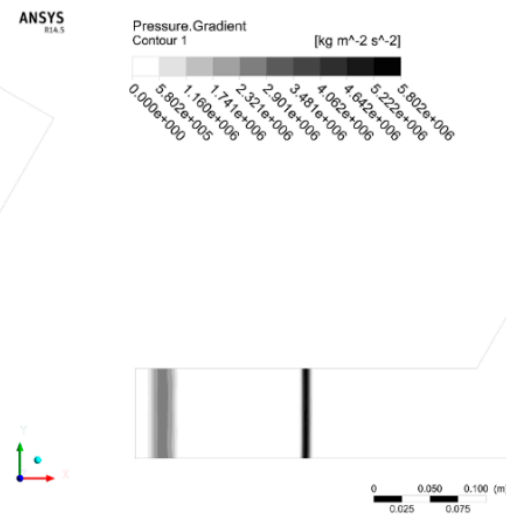

$\stackrel{\leftrightarrow}{\longrightarrow}$

(b)

ANSYS

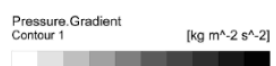

ANSYS

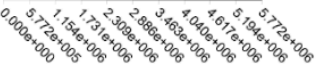

$\stackrel{\leftrightarrow}{\longrightarrow}$

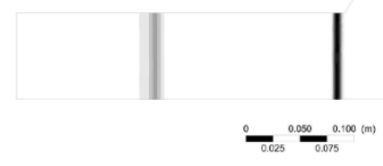

$\stackrel{\leftrightarrow}{\longrightarrow}$

Figure 9. Cont. 


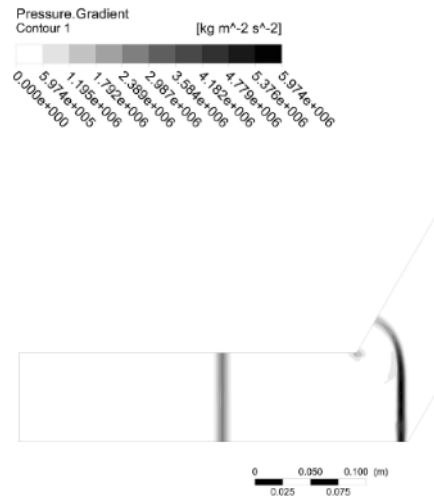

(e)
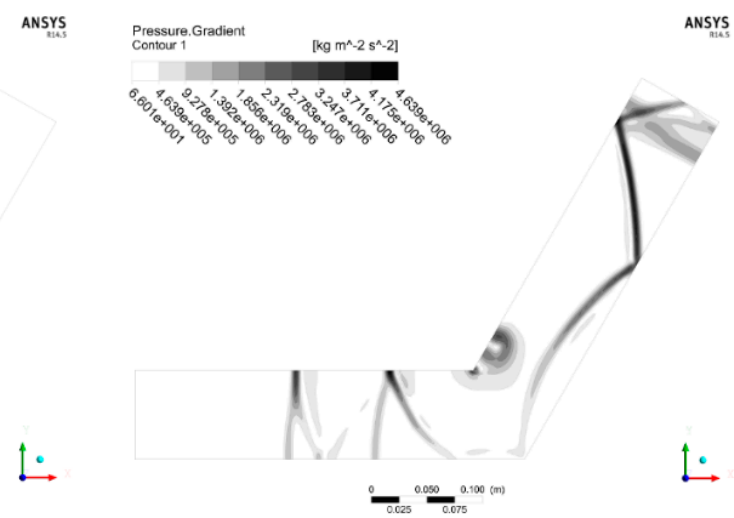

(f)

Figure 9. Overpressure gradient contours for blast-wave propagating through $120^{\circ}$ bend after (a) 25 time steps, (b) 100 time steps, (c) 200 time steps, (d) 300 time steps; and at (e) $0.000679 \mathrm{~s}$ and (f) $0.00158 \mathrm{~s}$.
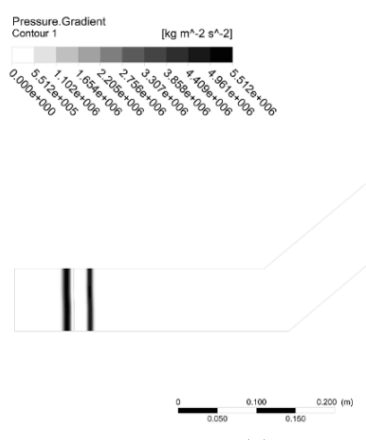

(a)
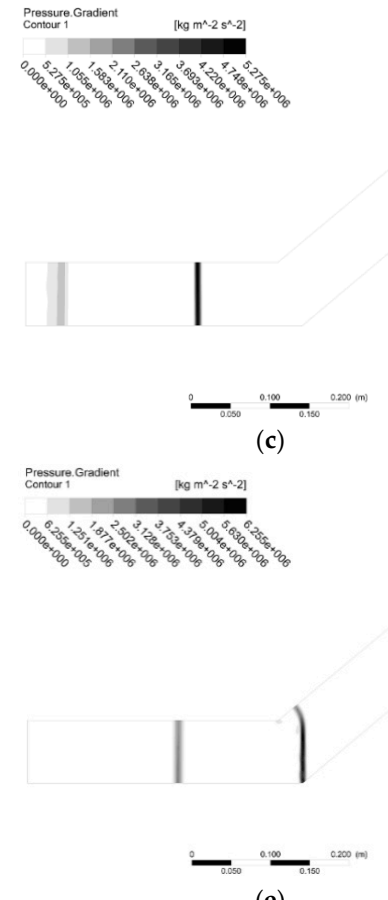

(e)
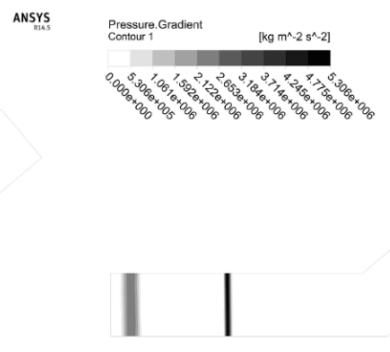

$\stackrel{\uparrow}{\longrightarrow}$

ansys

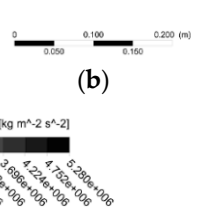

$\stackrel{\circ}{\circ}$

AnsYS

$\stackrel{\uparrow}{\perp}$

(d)

Ansys

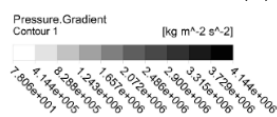

Ansys

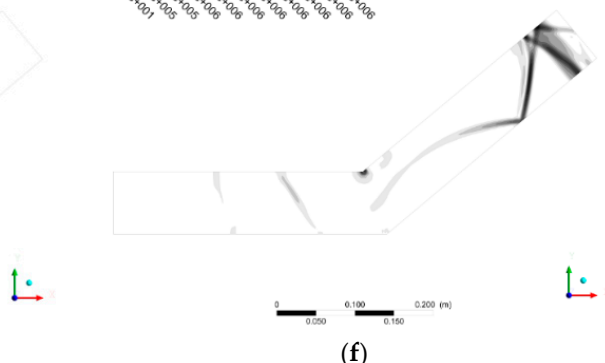

Figure 10. Overpressure gradient contours for blast-wave propagating through $140^{\circ}$ bend after (a) 25 time steps; (b) 100 time steps; (c) 200 time steps; (d) 300 time steps; and at (e) $0.000692 \mathrm{~s}$ and (f) $0.00164 \mathrm{~s}$. 


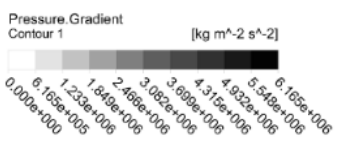

ANSYS

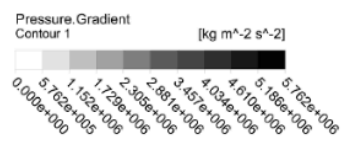

ANSYS
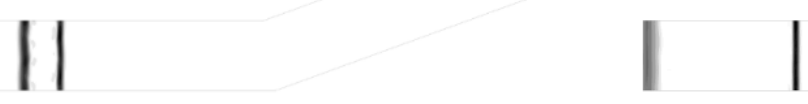

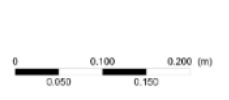

(a)
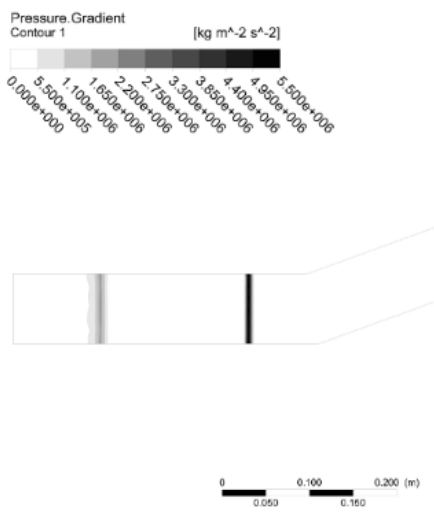

(c)
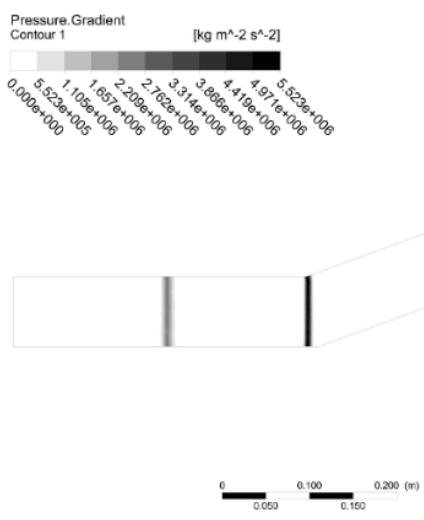

(e) $\stackrel{\leftrightarrow}{\longrightarrow}$

$\underset{\substack{\text { ANSYS } \\ \text { Hos }}}{ }$

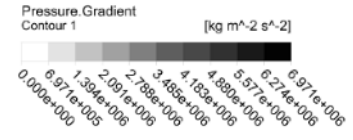

(b)

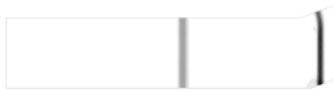

$\stackrel{\leftrightarrow}{\longrightarrow}$

$\underset{\text { ANSYS }}{\text { AMS }}$

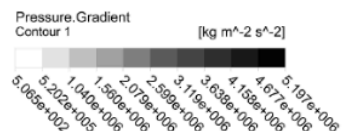

(d)

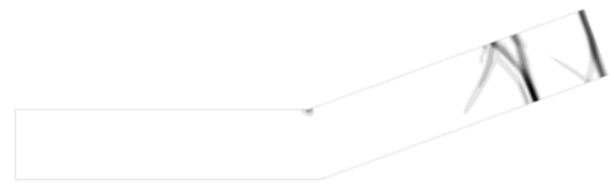

$\stackrel{\circ}{\longrightarrow}$<smiles>C=C[As]C</smiles>

$\stackrel{\leftrightarrow}{\longrightarrow}$

Figure 11. Overpressure gradient contours for blast-wave propagating through $160^{\circ}$ bend after (a) 25 time steps; (b) 100 time steps; (c) 200 time steps; (d) 300 time steps; and at (e) $0.000695 \mathrm{~s}$ and (f) $0.00159 \mathrm{~s}$.

The predictions of pressure gradient contours over the bends with angles of $40^{\circ}, 50^{\circ}, 90^{\circ}, 120^{\circ}$, $140^{\circ}$, and $160^{\circ}$ shows similar propagation patterns as the $30^{\circ}$ case. High pressure gradient regions are detected in the vicinity of the inner angle of the bends where a vortex forms. Incident waves propagate through the turn and then are reflected between the walls of the downstream gallery. Cases where the angle is greater than $90^{\circ}$ show a greater number of reflections and likely maintain longer peaks than acute angle cases. However, the reflected first shock front from the downstream leg becomes vaguer as the degree increases due to the incident waves and as the reflected waves are counteracted by each other, the pressure gradient vanishes gradually. 
Figure 12 provides a clear picture of the predicted upstream and downstream overpressure histories of bends with the angle of $40^{\circ}, 50^{\circ}, 90^{\circ}, 120^{\circ}, 140^{\circ}$, and $160^{\circ}$. As has been discussed before, the obtuse angle scenarios show a longer peak and the attenuation effect is not obvious (Figure 12d-f). For the $40^{\circ}, 50^{\circ}$, and $90^{\circ}$ cases, however, a sharp decrease of overpressure around $0.0016 \mathrm{~s}$ from the downstream monitor surface is observed.

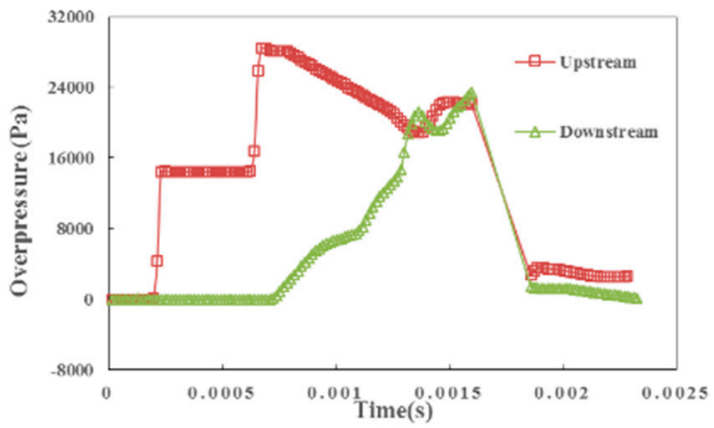

(a)

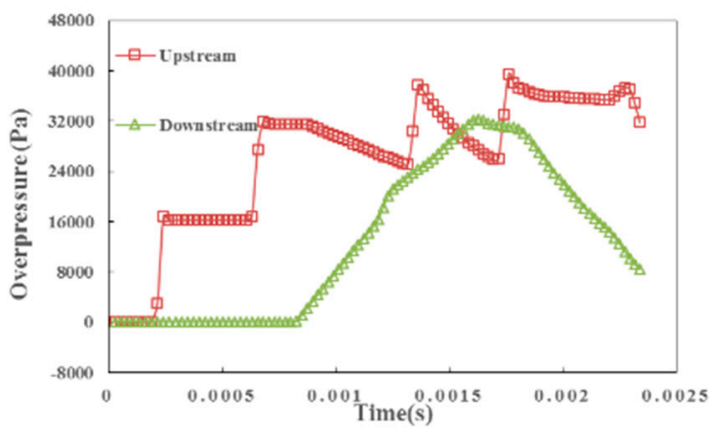

(c)

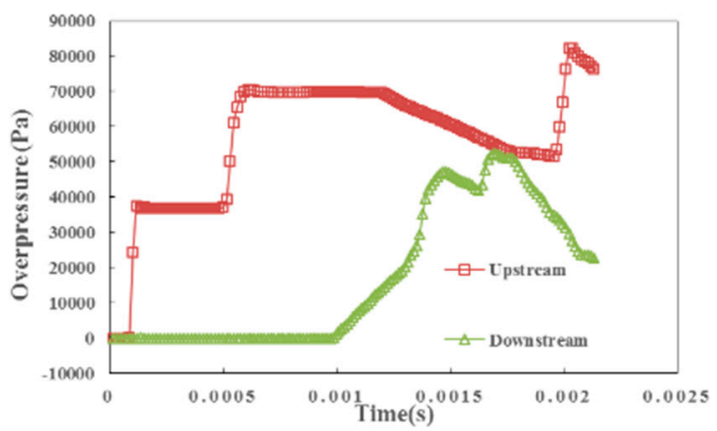

(e)

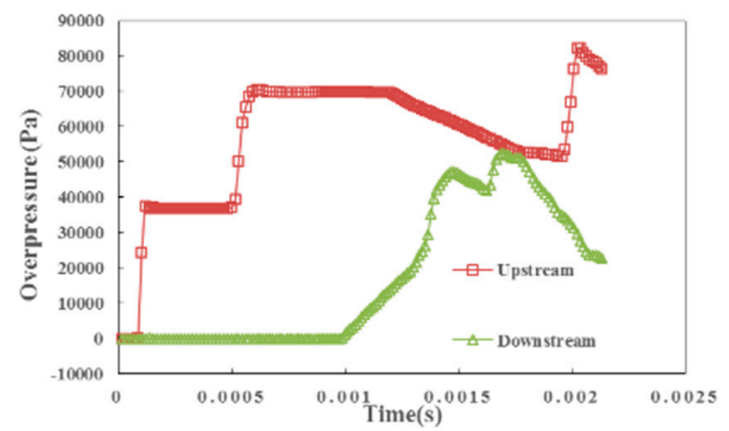

(b)

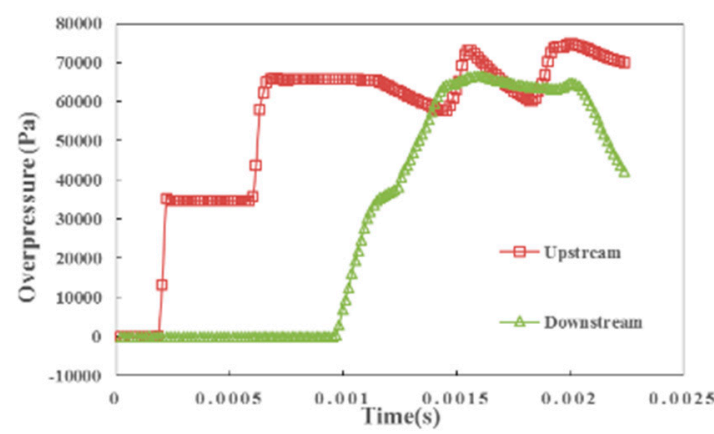

(d)

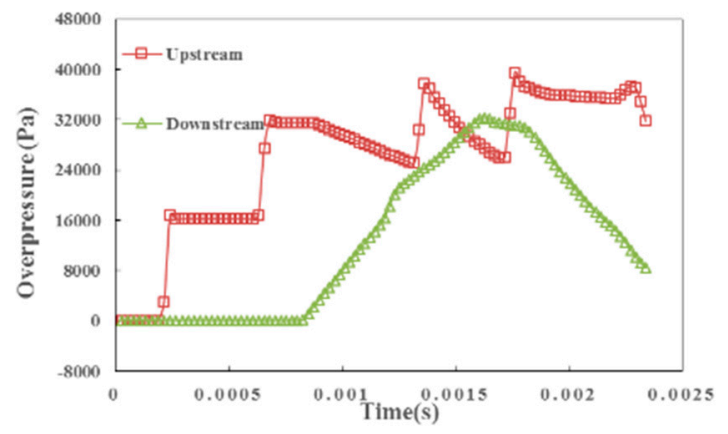

(f)

Figure 12. Overpressure histories upstream and downstream of (a) $40^{\circ}$; (b) $50^{\circ}$; (c) $90^{\circ}$; (d) $120^{\circ}$; (e) $140^{\circ}$; and (f) $160^{\circ}$ bends.

\subsection{Obstacles}

Blast-waves propagate through obstacles with blockage ratios (BR) of $25 \%, 50 \%$, and $75 \%$ are simulated and analyzed using the same strategy as the bend cases. BR is calculated as the blocked area divided by the total area of the cross-section. The total length of the selected gallery is $0.705 \mathrm{~m}$. The obstacle is located at $0.35 \mathrm{~m}$ downstream of the inlet and is $0.005 \mathrm{~m}$ thick. The location of the obstacle in the experimental explosion duct is shown in Figure 13g, right after the $9.45 \mathrm{~m}$ pre-duct shown in Figure 1. The diameter of the duct is also $0.08 \mathrm{~m}$. The pressure sensor upstream of the bend is placed along the centerline of the duct, $0.12 \mathrm{~m}$ apart from the inlet. The sensor downstream of the bend is placed along the duct centerline, $0.12 \mathrm{~m}$ from the outlet. 


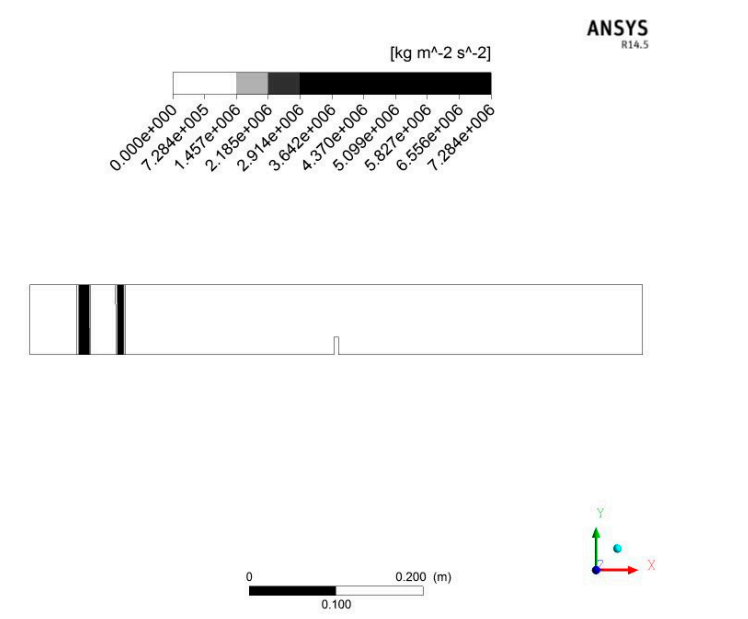

(a)
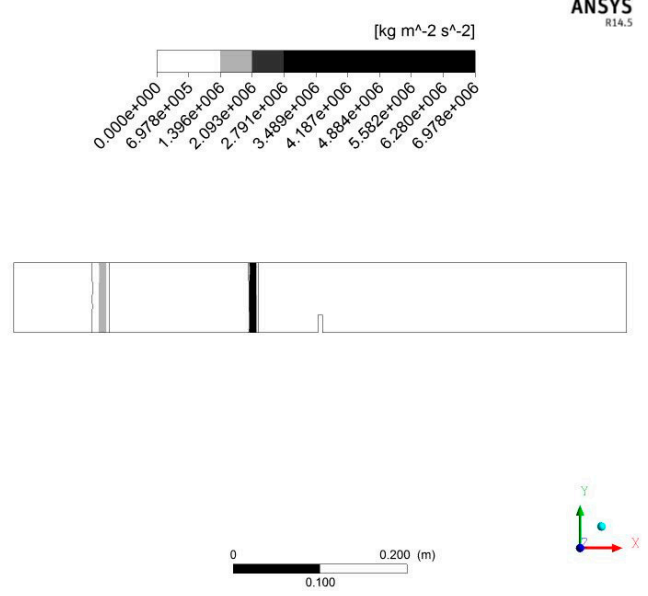

(c)

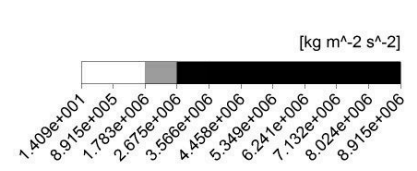

ANSYS
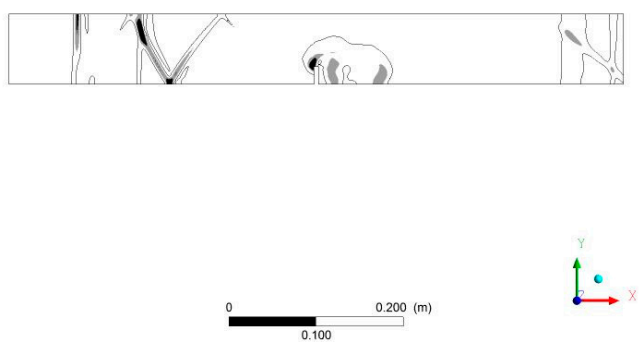

(e)

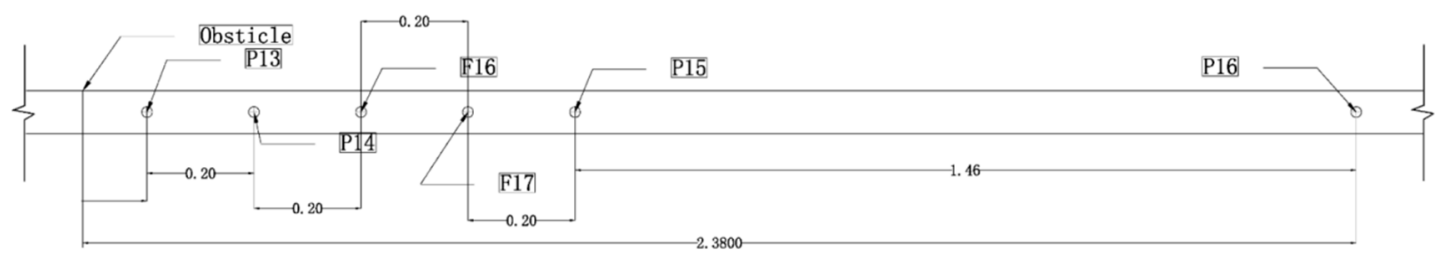

$(\mathrm{g})$

Figure 13. Overpressure gradient contours for the blast-wave propagating through obstacles with a BR of $25 \%$ after (a) 25 time steps; (b) 100 time steps; (c) 200 time steps; (d) 300 time steps; and at (e) $0.00183 \mathrm{~s}$ and (f) $0.00215 \mathrm{~s}$; (g) shows the location of the obstacle in experimental and numerical explosion ducts. 
BR 25\%. The predicted overpressure gradient contours after 25, 100, 200, and 300 time steps when the blast-wave was passing through an obstacle with a blockage ratio of $25 \%$ are shown in Figure $13 a-d$. Figure 13e,f show the overpressure contours at the instants of $0.00183 \mathrm{~s}$ and $0.00215 \mathrm{~s}$, when maximum overpressures of the first impulse were obtained by the upstream and downstream sensors.

Figure 14 shows the overpressure histories upstream and downstream of an obstacle with a BR of $25 \%$. The maximum overpressure for the first impulse upstream of the bend is $103,392 \mathrm{~Pa}$ at $0.00183 \mathrm{~s}$, while the attenuated maximum overpressure for the first impulse downstream the bend is $77,866 \mathrm{~Pa}$ at $0.00215 \mathrm{~s}$, the attenuation factor $\eta$ is 1.328 .

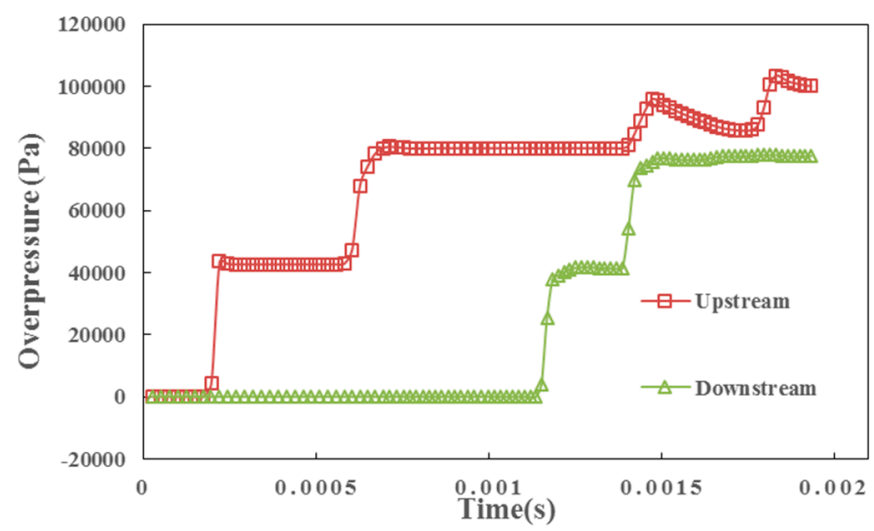

Figure 14. Overpressure histories upstream and downstream of BR 25\%.

BR $50 \%$ and $75 \%$. The overpressure gradient contours with blockage ratios of $50 \%$ and $75 \%$ are similar as the BR 25\% case and, therefore, will not be demonstrated. However, their attenuation capability varies significantly. The details will be discussed in Section 5 .

The comparison of overpressure histories between the BR 50\% and 75\% scenarios are shown in Figure 15. Similar to the BR 25\% case, the downstream overpressure maintains relatively high levels with the predicted $\eta$ of 1.559 and 1.959, respectively. This pattern of downstream overpressure histories is also seen in the bend cases with obtuse angles; however, obstacles provide more remarkable attenuation effects on a blast-wave propagation.

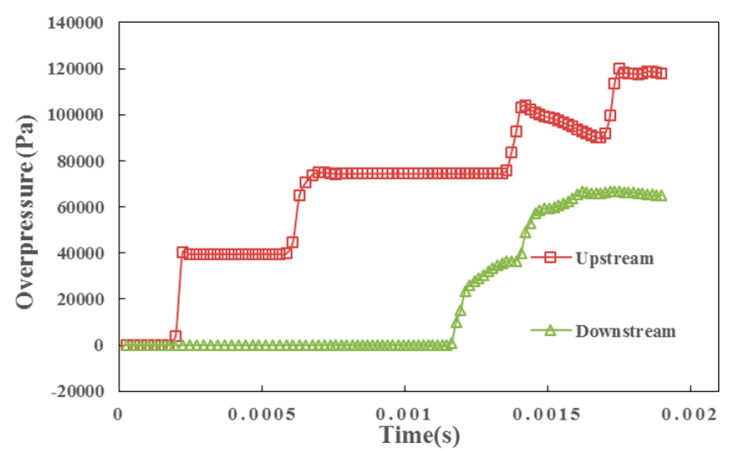

(a)

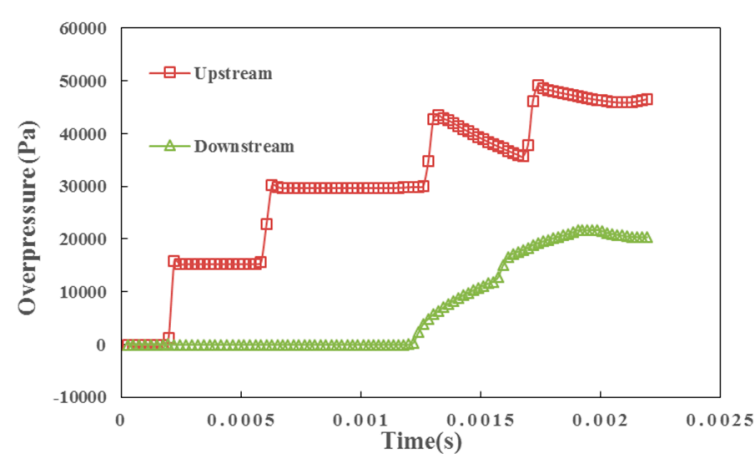

(b)

Figure 15. Overpressure histories upstream and downstream of (a) BR 50\% and (b) BR 75\%.

\subsection{T-Branches}

The last category of geometric changes selected for this study include two T-branch cases. The length of the three arms, perpendicular to each other, of the T-branch is $0.35 \mathrm{~m}$. The diameter of the duct is $0.08 \mathrm{~m}$. The pressure sensor upstream of the T-branch was placed along the centerline of 
the duct, $0.12 \mathrm{~m}$ apart from the inlet. The sensors downstream were placed along the duct centerline, $0.12 \mathrm{~m}$ from the outlet.

The predicted overpressure gradient contours, and where it flowed from the main arm to the branch arm, are shown in Figure 16 when maximum overpressures of the first impulse were obtained by the downstream sensors.

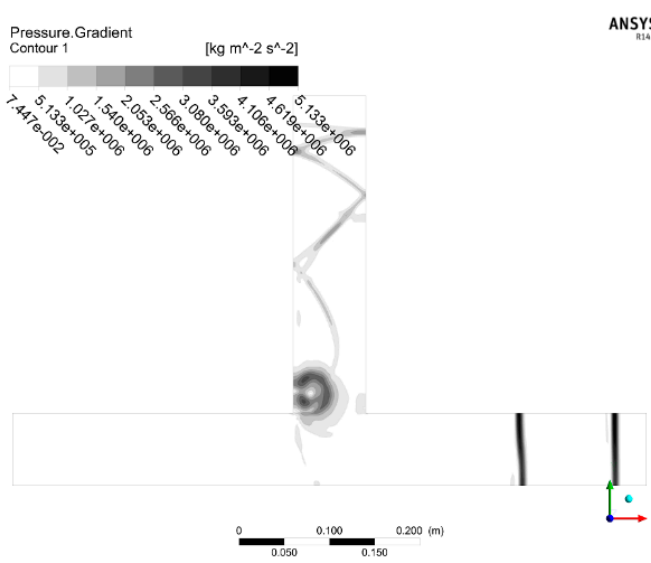

(a)

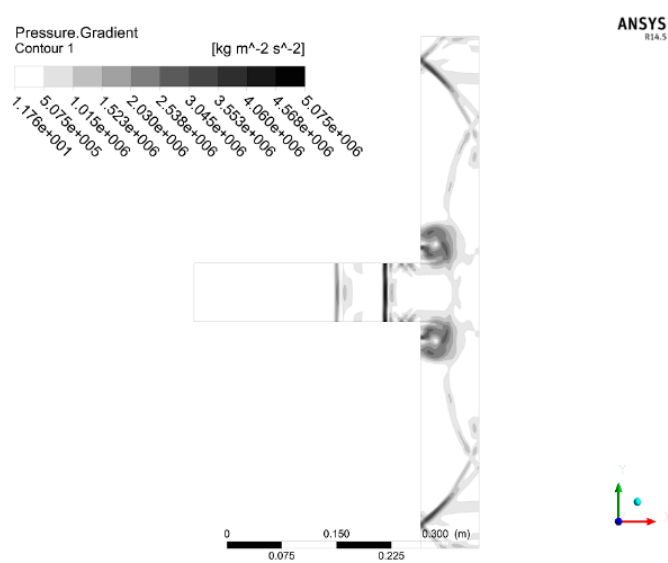

(b)

Figure 16. Overpressure gradient contours for a blast-wave propagating through the T-branch (a) flow from the branch arm and (b) flow from the main arm when the downstream sensors reach maximum overpressures.

The overpressure histories upstream and downstream of the two T-branch scenarios are demonstrated in Figure 17.

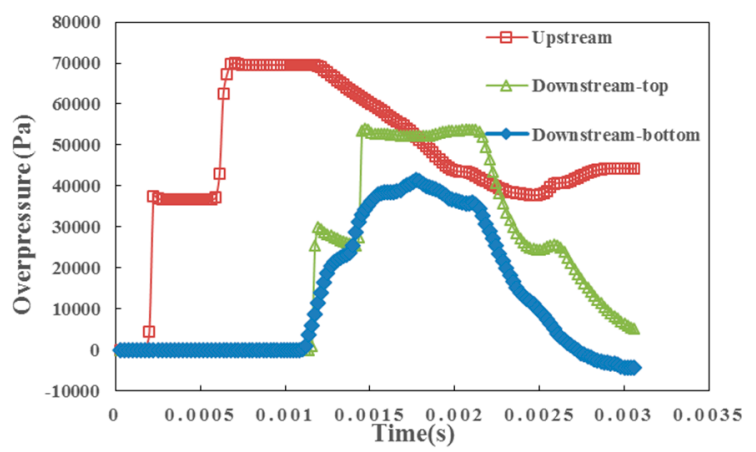

(a)

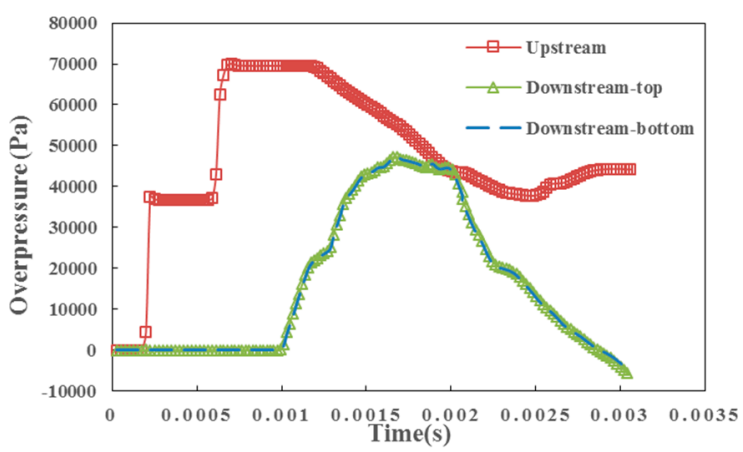

(b)

Figure 17. Overpressure histories upstream and downstream of the T-branch flows from the (a) main arm and (b) branch arm.

For the case where the incident wave propagates from the main arm (Figure 17a), the maximum overpressure for the first impulse upstream of the bend was $69,651 \mathrm{~Pa}$ at $0.000658 \mathrm{~s}$, while the attenuated maximum overpressure for the first impulse of the downstream-top and downstream-bottom were $41,383 \mathrm{~Pa}$ at $0.00177 \mathrm{~s}$ and $53,974 \mathrm{~Pa}$ at $0.00147 \mathrm{~s}$. The attenuation factor $\eta$ for the top branch is 1.683 while for the bottom main arm is 1.29. For the other case, the upstream and downstream overpressure histories are demonstrated in Figure $17 \mathrm{~b}$ with the overpressure gradient contour shown in Figure 16b. In this case, the maximum overpressure for the first impulse upstream the bend is 70,037 $\mathrm{Pa}$ at $0.000702 \mathrm{~s}$ and the attenuated maximum overpressure for the first impulse of the downstream-top and downstream-bottom are $47,353 \mathrm{~Pa}$ at $0.00171 \mathrm{~s}$ and $473,285 \mathrm{~Pa}$ at $0.00171 \mathrm{~s}$, respectively. Attenuation factors $\eta$ for the top and bottom branches are 1.479 and 1.481, which are almost identical due to 
its symmetrical shape. The case of incident wave input from the branch arm is validated by the experimental data, which present $\eta$ values of 1.86 (predicted as 1.683) and 1.344 (predicted as 1.29) for the top and bottom arms, respectively. The accuracy of the numerical prediction is acceptable.

The attenuation effect of bend and obstacle cases on the overpressure of the blast-wave will be discussed in the next section. As there is an absence of experimental data for T-branch cases when the incident wave is induced from the main arm, this case will be excluded from further discussion.

\section{Discussions}

\subsection{Bends}

The influences of the geometric changes on the way a blast-wave propagates is quantified by attenuation factors $\eta$. The predicted and measured $\eta$ of all bends are listed in Table 2. Bends with angles of $30^{\circ}, 40^{\circ}$, and $160^{\circ}$ are not covered by the experiment in this study.

Table 2. Predicted and experimental attenuation factors for bends.

\begin{tabular}{cccc}
\hline Bending & Predicted $\boldsymbol{\eta}$ & Experimental $\boldsymbol{\eta}$ & Relative Error (\%) \\
\hline $30^{\circ}$ & 1.150 & $\mathrm{Na}$ & $\mathrm{Na}$ \\
$40^{\circ}$ & 1.214 & $\mathrm{Na}$ & $\mathrm{Na}$ \\
$50^{\circ}$ & 1.534 & 1.726 & 11.12 \\
$90^{\circ}$ & 1.140 & 1.131 & 0.80 \\
$120^{\circ}$ & 0.991 & 0.703 & 40.97 \\
$140^{\circ}$ & 0.995 & 0.945 & 5.29 \\
$160^{\circ}$ & 1.002 & $\mathrm{Na}$ & $\mathrm{Na}$ \\
\hline
\end{tabular}

Na refers to not available.

Based on the records in Table 2, the comparison of predicted and experimental values are shown in Figure 18. The red curve represents simulation results while the blue one represents measured data.

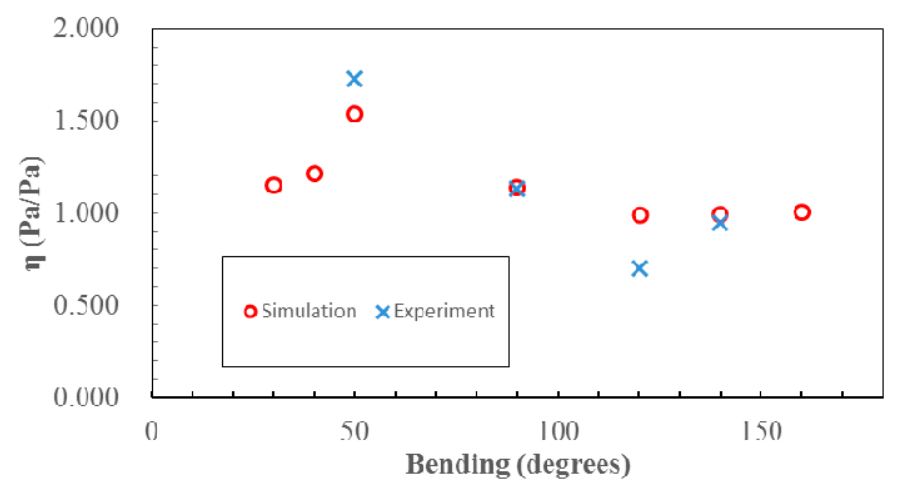

Figure 18. Comparison of simulation and experimental attenuation factors for the bends.

As illustrated in Figure 18, the predicted $\eta$ is in reasonable agreement with experimental values in the cases of $50^{\circ}, 90^{\circ}$, and $150^{\circ}$ bends, with relative errors of $0.8 \%, 5 \%$, and $11 \%$, respectively. In the $120^{\circ}$ case, however, the prediction overestimates the attenuation factor by $41 \%$. In the discussion of experimental results, it was explained that the bend with a $120^{\circ}$ angle tends to strengthen the blast-wave further than predicted. However, this strengthening effect is not observed at the level of $140^{\circ}$. This observable difference might be due to leakage during the experiment and shear heating for the $120^{\circ}$ case, which is neglected in the numerical simulation. This effect is weakened in the $140^{\circ}$ case due to a slight change of the wave propagating direction. 


\subsection{Obstacles}

Attenuation factors of three selected obstacle scenarios with different blockage ratios from experiments and simulations are listed in Table 3 and plotted in Figure 19.

As seen in both Table 3 and Figure 19, the predicted values are commonly greater than the experimental by around $15 \%$. This might be due to the ideal blast-wave simplification applied in the numerical simulations that ignores the overpressure decrement after the first impulse. To use this model for predicting more BR cases, a 15\% adjustment factor should be applied on the each of the simulation results.

Table 3. Predicted and experimental attenuation factors for obstacles with three BRs.

\begin{tabular}{cccc}
\hline BR (\%) & Predicted $\boldsymbol{\eta}$ & Experimental $\boldsymbol{\eta}$ & Relative Error (\%) \\
\hline 25 & 1.329 & 1.1 & 20.82 \\
50 & 1.559 & 1.326 & 17.57 \\
75 & 1.959 & 1.759 & 11.37 \\
\hline
\end{tabular}

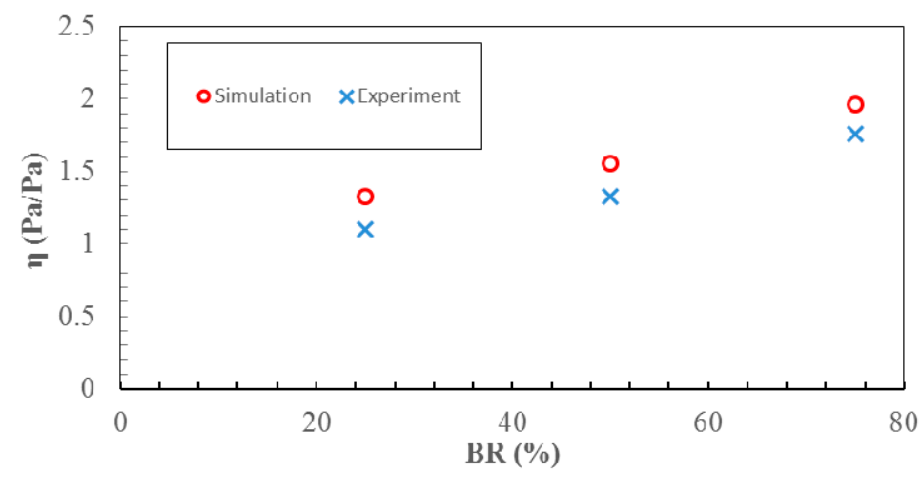

Figure 19. Comparison of simulation and experimental attenuation factors for obstacles.

\section{Conclusions}

In this study, the attenuation effect of seven types of bends, three obstacles, and two T-branches are investigated numerically. Three main conclusions have been drawn from the preceding analysis: (1) as a bending angle increases from $0^{\circ}$ to $180^{\circ}$ the attenuation factor increases at $30^{\circ}$ until the maximum value is obtained at $50^{\circ}$. Then, it decreases to around 1.14 in the $90^{\circ}$ case. The bends with angles greater than $90^{\circ}$ tend to slightly strengthen blast-waves; (2) for the obstacles with different blockage ratios, the attenuation factor increases as BR increases; (3) the compressible, and unsteady numerical Euler scheme used in this research tends to overestimate the attenuation factors in the $120^{\circ}$ bend; and the obstacles with BR $25 \%$, BR 50\%, and BR 75\% were in reasonable agreement with experimental results.

The attenuation factor is a rudimentary indicator of the influence of a geometric change, but it has been suggested that the overpressure history curves and its trend should also be considered when dealing with practical problems. Numerical models are simplifications of real cases; the predictions of this research that deviated from the experimental values should be used with extra care. The results of this work could be applied in global overpressure distribution prediction for gas explosions occuring in a full-size underground airway network if the fluid dynamic parameters and the gallery dimensions are reasonably justified.

Acknowledgments: This work is financially supported by grants from the National Natural Science Foundation of China (Grant No. 51504045), College Foundation of science and technology innovations (Grant No. 106112016CD JXY24005). Gas Explosion Lab of China University of Mining and Technology provided the platform for experimental study.

Author Contributions: Liang Wang did experiment and built numerical simulation; Sisi Que analyzed experimental data; Jerry Tien and Nassib Aouad designed experiment. 
Conflicts of Interest: The authors declare no conflict of interest.

\section{References}

1. McPherson, M.J. Subsurface Ventilation Engineering; Mine Ventilation Services: Clovis, CA, USA, 1993; Chapter 21; pp. 45-46.

2. Brnich, M.J.; Kowalski-Trakofler, K.M. Mining Publication: Underground Coal Mine Disasters 1900-2010: Events, Responses, and a Look to the Future; Society of Mining, Metallurgy, and Exploration: Englewood, CO, USA, 2010; pp. 363-372.

3. Chen, X.F.; Zhang, Y. Effect of $\mathrm{CH}_{4}$-Air Ratios on Gas Explosion Flame Microstructure and Propagation Behaviors. Energies 2012, 5, 4132-4146. [CrossRef]

4. Leisenheimer, B.; Leuckel, W. Self-Generated Acceleration of Confined Deflagrative Flame Fronts. Combust. Sci. Technol. 1996, 118, 147-164. [CrossRef]

5. Zhang, L.; Zhang, Y.; Xu, J.; Yang, G.; Hou, S.; Yang, L. Numerical Simulation of Shock Wave Structure in Gas Explosion. Procedia Eng. 2011, 26, 1322-1329.

6. Jia, Z.W.; Liu, Y.W.; Jin, X.G. Propagation Characteristic about Shock Wave of Gas Explosion at Laneway Corner. J. China Coal Soc. 2011, 36, 97-100.

7. Kordylewski, W.; Wach, J. Influence of Ducting on Explosion Pressure: Small Scale Experiments. Combust. Flame 1988, 71, 51-61. [CrossRef]

8. Lin, B.Q.; Zhou, S.N.; Zhang, R.G. Influence of Barriers on Flame Transmission and Explosion Wave in Gas Explosion. J. China Univ. Min. Technol. 1999, 28, 104-106.

9. Lin, B.Q.; Zhu, C.J. The Influences of Bend on Methane Explosion Propagation in Underground Coal Mines. In Proceedings of the China National Occupational Safety and Health Association Annual Conference, Beijing, China, 28-30 October 2009; pp. 312-318.

10. Xu, J.D.; Yang, G.Y. Numerical Simulation of the Barricade Encouraging Effect in Process of Gas Explosion Propagation. China Saf. Sci. J. 2003, 13, 42-44.

11. Lin, B.Q.; Ye, Q.; Zhai, C.; Jian, C.G. The propagation rule of methane explosion in bifurcation duct. J. China Coal Soc. 2008, 2, 136-139.

12. Zhou, X.Q.; Wu, B.; Xu, J.D. Basic Characters of Gas Explosion in Underground Coal Mines. China Coal Mines 2002, 28, 8-11.

13. Wang, L.; Que, S.; Tien, J.C.; Aouad, N.S. Scale Effect of Premixed Methane-Air Combustion in Confined Space Using LES Model. Minerals 2016, 6. [CrossRef]

14. Hjertager, B.H. Computer Modelling of Turbulent Gas Explosion in Complex 2D and 3D Geometries. J. Hazard. Mater. 1993, 34, 173-197. [CrossRef]

15. Dai, L.C.; Zhao, C.H.; Liu, Z.; Hu, T.Z. Numerical Simulation Study of Gas Explosion Propagation Law in the Pipe. Min. Eng. Res. 2011, 26, 31-32.

16. ANSYS, Inc. ANSYS FLUENT Theory Guide; ANSYS, Inc.: Canonsburg, PA, USA, 2011.

(C) 2016 by the authors; licensee MDPI, Basel, Switzerland. This article is an open access article distributed under the terms and conditions of the Creative Commons Attribution (CC-BY) license (http://creativecommons.org/licenses/by/4.0/). 